\title{
Defining asthma-COPD overlap syndrome: a population-based study
}

\author{
Tobias N. Bonten ${ }^{1,2}$, Marise J. Kasteleyn ${ }^{1,2}$, Renee de Mutsert ${ }^{3}$, \\ Pieter S. Hiemstra1, Frits R. Rosendaal ${ }^{3,4}$, Niels H. Chavannes ${ }^{2}$, \\ Annelies M. Slats ${ }^{1}$ and Christian Taube ${ }^{1}$
}

Affiliations: ${ }^{1}$ Dept of Pulmonology, Leiden University Medical Center, Leiden, The Netherlands. ${ }^{2}$ Dept of Public Health and Primary Care, Leiden University Medical Center, Leiden, The Netherlands. ${ }^{3}$ Dept of Clinical Epidemiology, Leiden University Medical Center, Leiden, The Netherlands. ${ }^{4}$ Dept of Thrombosis and Haemostasis, Leiden University Medical Center, Leiden, The Netherlands.

Correspondence: T.N. Bonten, Dept of Pulmonology, Leiden University Medical Center, V6-22, P0 Box 9600, 2300 RC Leiden, The Netherlands. E-mail: t.n.bontendlumc.nl

@ERSpublications

Agreement between ACOS definitions is low, and prevalence and exacerbation risk depends strongly on the definition http://ow.ly/FR8i3089Q27

Cite this article as: Bonten TN, Kasteleyn MJ, de Mutsert R, et al. Defining asthma-COPD overlap syndrome: a population-based study. Eur Respir J 2017; 49: 1602008 [https://doi.org/10.1183/ 13993003.02008-2016].

ABSTRACT Asthma-chronic obstructive pulmonary disease (COPD) overlap syndrome (ACOS) seems an important clinical phenotype, but multiple definitions have been proposed. This study's objectives were to assess the effect of different ACOS definitions on prevalence, patient characteristics and exacerbations.

5675 individuals aged 45-65 years, with 846 asthma/COPD patients, were included in the Netherlands Epidemiology of Obesity study between 2008 and 2012, and followed-up for a median of 1.8 years. ACOS was defined by recent consensus criteria and five other definitions, based on registry, questionnaires and lung function.

Prevalence of ACOS in the asthma/COPD population ranged between $4.4 \%$ and $38.3 \%$, depending on the definition used. Agreement between registry-based and self-reported ACOS was 0.04 and 0.41 when lung function (forced expiratory volume in $1 \mathrm{~s}(\mathrm{FEV} 1) /$ forced vital capacity $(\mathrm{FVC})<0.7)$ was added. With registry or self-report defined ACOS, only $51 \%$ and $33 \%$ had FEV1/FVC $<0.7$. Patient characteristics were similar, but asthma duration was longer with self-reported compared with registry-based ACOS (mean difference 22 years (95\% CI 12-33)). Exacerbation risk was highest with registry-based ACOS compared with asthma (adjusted incidence rate ratio 1.6 (95\% CI 1.2-2.1)).

This study adds important knowledge about agreement between ACOS definitions and their relation with exacerbations. Given the low agreement, differences in prevalence, patient characteristics and risk of exacerbations, consensus about ACOS definition in different care settings is urgently needed.

This article has supplementary material available from erj.ersjournals.com

Received: Oct 132016 | Accepted after revision: Jan 032017

Support statement: The Netherlands Epidemiology of Obesity (NEO) study is supported by the participating departments, the Division and the Board of Directors of the Leiden University Medical Center, and by the Leiden University, Research Profile Area "Vascular and Regenerative Medicine".

Conflict of interest: None declared.

Copyright OERS 2017 


\section{Introduction}

Asthma and chronic obstructive pulmonary disease (COPD) affect $\sim 10 \%$ of the population $[1,2]$. Asthma and COPD are considered different disease entities. However, in everyday practice, patients may experience symptoms of both asthma and COPD. The presence of asthma and COPD symptoms in the same patient has recently been named asthma-COPD overlap syndrome (ACOS) [3]. Following the publication of a key article in 2009 [4], this entity has been put forward by international asthma associations and recommendations, such as the Global Initiative for Asthma (GINA) and the Global Initiative for Chronic Obstructive Lung Disease (GOLD). These documents offer no definition for ACOS but rather a description, stating that ACOS is characterised by persistent airflow limitation with several features usually associated with asthma and several features usually associated with COPD [3]. However, a clear definition of ACOS is lacking and therefore it is an important challenge to adequately identify patients with ACOS. Indeed, multiple definitions have been proposed by different entities and countries [3,5-8]. Recently, a consensus definition was proposed by an international committee of experts [9]. However, the definitions used in the current available scientific literature are inconsistent, making comparison between studies a major challenge. For example, some studies used doctor-diagnosed COPD and asthma, whereas other studies used self-reported COPD and asthma to define ACOS [10]. Given differences in the definitions used in the scientific literature, there is a need to compare these definitions and their association with clinical end-points. Furthermore, prevalence and patient characteristics were studied in heterogeneous patient populations, varying from a smoking COPD-only population to an asthma-only and general population [10]. These variations in ACOS definition have even led previous authors to classify ACOS as "CAOS" and "useless" as a disease entity [11, 12]. Still, the clinical relevance of ACOS has been demonstrated by previous studies, showing that ACOS patients are at higher risk for exacerbations [13]. Thus, research on the effect of various ACOS definitions on patient characteristics and clinical outcomes is urgently needed. Therefore, we conducted a cohort study in the general population with an asthma/COPD subpopulation to assess the impact of different ACOS definitions on prevalence, patient characteristics and exacerbation risk.

\section{Materials and methods \\ Study subjects and design}

The Netherlands Epidemiology of Obesity (NEO) study is a population-based, prospective cohort study of 6671 individuals aged 45-65 years, with an oversampling of individuals with a body mass index (BMI) $\geqslant 27 \mathrm{~kg} \cdot \mathrm{m}^{-2}$. The study design and data collection have been described previously [14]. During the first stage, men and women living in the greater area of Leiden (in the west of the Netherlands) were invited by letters sent by general practitioners and municipalities and by local advertisements. They were invited to respond if they were aged between 45 and 65 years and had a self-reported BMI $\geqslant 27 \mathrm{~kg} \cdot \mathrm{m}^{-2}$. In addition, during a second stage, all inhabitants aged between 45 and 65 years from one municipality (Leiderdorp) were invited to participate, irrespective of their BMI, allowing a reference distribution of BMI. Participants were invited for a baseline visit at the NEO study centre of the Leiden University Medical Center (LUMC) after an overnight fast. Prior to this study visit, participants completed a general questionnaire at home to report demographic, lifestyle and clinical information. All participants underwent an extensive physical examination, including anthropometry and spirometry. In total, 6671 participants were included between 2008 and 2012. In 2013, medical history and medication prescription was obtained through the electronic medical record (EMR) registry of their general practitioner. International Classification of Primary Care (ICPC) codes and medication use (Anatomical Therapeutic Chemical Classification System) during follow-up were extracted for 6442 (97\%) participants. The Medical Ethical Committee of the LUMC approved the design of the study and all participants gave their written informed consent.

\section{Methods}

Participants reported smoking habits by questionnaire, categorised into current smokers, ex-smokers or never (reference)-smokers. Physical activity was reported using the SQUASH (short questionnaire to assess health-enhancing physical activity) questionnaire and expressed in metabolic equivalents of task hours during leisure time per week [15]. Participants reported the type, duration and daily dose of medication use, including inhalation medication. Additionally, participants were asked to bring all medications they were using at that time to the study visit, which were checked and recorded by study nurses. Furthermore, participants reported their medical history of cardiovascular disease (CVD). Pre-existing CVD was defined as myocardial infarction, angina, congestive heart failure, stroke or peripheral vascular disease. The presence of diabetes was defined as self-report of diabetes or use of glucose-lowering medication. The presence of depression was defined according to the Inventory of Depressive Symptomatology (IDS) questionnaire as a total score $\geqslant 18$ [16]. This cut-off has a sensitivity of $94 \%$ to detect any depression [17]. Severe depression was defined as a score $\geqslant 29$ on the IDS questionnaire [16, 17]. At baseline visit, height 
and weight were measured without shoes and with a precision of $0.1 \mathrm{~cm} \cdot \mathrm{kg}^{-1}$, and $1 \mathrm{~kg}$ was subtracted to correct for the weight of clothing. BMI was calculated by dividing the weight by the height squared $\left(\mathrm{kg} \cdot \mathrm{m}^{-2}\right)$. Waist circumference was measured with a horizontally placed tape measure mid-way between the lower costal margin and the iliac crest with a precision of $0.1 \mathrm{~cm}$. Hip circumference was measured at the maximum circumference of the buttocks. The waist/hip ratio (WHR) was calculated by dividing waist circumference by hip circumference.

\section{Pulmonary function tests}

Pulmonary function tests were performed at the Dept of Pulmonology at the LUMC. Spirometry was performed according to the standards of the European Respiratory Society [18]. A spirometer (Jaeger Masterscreen PFT; Viasys Healthcare, Hoechberg, Germany) was used by trained technicians to measure forced expiratory volume in $1 \mathrm{~s}(\mathrm{FEV} 1)$ and forced vital capacity (FVC). FEV $1 \%$ pred was defined as the FEV 1 value expressed as a percentage of the predicted normal FEV1 and FVC \% pred was defined as the FVC value expressed as a percentage of the predicted normal FVC, both predictions based on sex, age and height. The Tiffeneau index was calculated by dividing the registered FEV 1 value by the registered FVC value. Exhaled nitric oxide fraction $\left(F_{e N O}\right)$ was measured once with a NIOX MINO (Aerocrine, Solna, Sweden).

\section{Exacerbations}

Exacerbations were defined as doctor prescription of airway-related antibiotics (amoxicillin (-clavulanic acid), doxycycline, azithromycin, erythromycin), oral corticosteroids (30-40 mg) or both, use of which is indicative for a moderate exacerbation [19].

\section{Asthma, COPD and ACOS definitions}

Asthma and COPD were defined by self-reporting in baseline questionnaires or by ICPC codes in the primary care EMR (codes R95 and R96). ACOS was defined according to the presence of Asthma and COPD in the EMR, questionnaires, and lung function (FEV1/FVC and FeNO), or a combination of these. As no specific registry code is available for ACOS in the Netherlands, we additionally defined ACOS if the general practitioner used ACOS-specific text words in medical records (supplementary table S1). For the present analyses six definitions of ACOS were used: 1) COPD AND Asthma in registry; 2) COPD AND Asthma in registry OR ACOS as text in EMR; 3) self-reported COPD AND Asthma; 4) three major consensus criteria: FEV1/FVC $<0.7$ AND at least 10 pack-years AND asthma before 40 years of age [9]; 5) COPD in registry OR Self-reported AND FEV1/FVC $<0.7$ AND Asthma in registry OR Self-reported; and 6) COPD in registry OR Self-reported OR FEV1/FVC $<0.7$ AND Asthma in registry OR Self-reported OR $F \mathrm{eNO} \geqslant 45 \mathrm{ppb}[6,20]$.

\section{Analysis}

In the NEO study individuals with a $\mathrm{BMI} \geqslant 27 \mathrm{~kg} \cdot \mathrm{m}^{-2}$ were oversampled. To correctly represent associations in the general population, adjustments for oversampling of individuals with a BMI $\geqslant 27 \mathrm{~kg} \cdot \mathrm{m}^{-2}$ were made [21]. This was done by weighting the participants towards the BMI distribution of participants from the Leiderdorp municipality, whose BMI distribution was similar to the BMI distribution of the general Dutch population. All baseline results were based on weighted analyses and consequently apply to a population-based study without oversampling of participants with a BMI $\geqslant 27 \mathrm{~kg} \cdot \mathrm{m}^{-2}$ [22]. Baseline characteristics were expressed as mean $\pm \mathrm{sD}$, median (interquartile range (IQR)) or proportion (\%). From the total study population, participants with missing data were excluded, thus yielding a complete case study population. Within this complete case study population, a subpopulation of asthma and/or COPD patients was selected, based on the presence of asthma or COPD in medical registries and questionnaires. The prevalence of ACOS, according to different definitions, was calculated in the complete case study population and the asthma/COPD subpopulation. Differences in patient characteristics between definitions were assessed with linear regression for continuous outcomes or Chi-squared tests and logistic regression analysis for categorical outcomes, with definition 1 as the reference category. Agreement between the ACOS definitions was calculated with the $\kappa$ statistic and plotted in a Venn diagram. Exacerbation rates of ACOS patients according to the six definitions were compared with asthma and COPD patients during follow-up using Poisson regression and reported as IRRs. Analyses were performed with STATA version 12.0 (Statacorp, College Station, TX, USA).

\section{Results}

Baseline data were missing for spirometry $(\mathrm{n}=201), \mathrm{FeNO}(\mathrm{n}=379)$, physical activity $(\mathrm{n}=121)$, self-reported asthma and COPD $(n=36)$, diabetes $(n=64)$, self-reported CVD $(n=28)$, IDS $(n=16)$, WHR $(n=8)$, smoking $(n=8)$, and number of pack-years $(n=147)$. A total of $238(4 \%)$ participants were lost to follow-up. 
Therefore, the present analyses were conducted in a complete case study population of 5675 participants. A subpopulation of 846 (15\%) participants with asthma and/or COPD was identified.

\section{Baseline characteristics}

The characteristics of the complete case study population are presented in table 1 . The majority (62\%) of participants were ex-smokers or current smokers. $15 \%$ of the participants had FEV1/FVC $<0.7$. Prevalence of COPD was lower in registry data than self-reported (2.5\% versus $4.2 \%)$. In contrast, asthma prevalence was higher in registry data than self-reported (8.0\% versus $4.4 \%)$. In the subpopulation of 846 asthma and/or COPD patients, the most frequently used medication was a long-acting $\beta$-agonist plus inhaled corticosteroid (ICS) combination (29\%), followed by short-acting $\beta$-agonist (15\%) and ICS (14\%) monotherapy. The most frequent comorbidity in the total population was depression (14\%).

\section{ACOS prevalence and agreement between definitions}

Prevalence of ACOS in the complete case study population and the asthma/COPD subpopulation is shown in table 2. In both populations, ACOS prevalence was not affected by identifying ACOS cases in the EMR

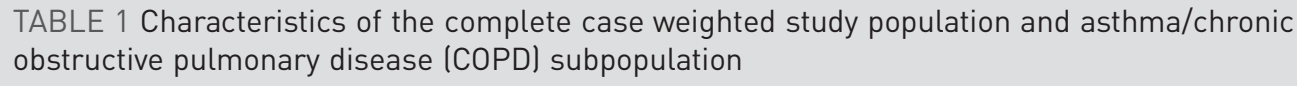

\begin{tabular}{|c|c|c|}
\hline & Total population & Asthma/COPD population \\
\hline Subjects n & 5675 & 846 \\
\hline Age years & $56 \pm 6$ & $56 \pm 6$ \\
\hline Male & 44 & 36 \\
\hline $\mathrm{BMI} \mathrm{kg} \cdot \mathrm{m}^{-2}$ & $26 \pm 4$ & $27 \pm 5$ \\
\hline Waist/hip ratio & $0.89 \pm 0.08$ & $0.89 \pm 0.09$ \\
\hline Physical activity MET-week ${ }^{-1}$ & $30(16-50)$ & $31(16-47)$ \\
\hline \multicolumn{3}{|l|}{ Smoking } \\
\hline Never & 38 & 33 \\
\hline Ex & 46 & 50 \\
\hline Current & 16 & 17 \\
\hline \multicolumn{3}{|l|}{ Lung disease } \\
\hline COPD, registry & 2.5 & 21.5 \\
\hline COPD, questionnaire & 4.2 & 35.6 \\
\hline Asthma, registry & 8.0 & 66.9 \\
\hline Asthma, questionnaire & 4.4 & 37.4 \\
\hline $\mathrm{FEV}_{1} / \mathrm{FVC}<0.7$ & 14.8 & 32.5 \\
\hline \multicolumn{3}{|l|}{ Lung medication use } \\
\hline Any bronchodilator & 5.3 & 40.2 \\
\hline SABA & 2.1 & 15.3 \\
\hline LABA & 0.4 & 3.1 \\
\hline SAMA & 0.1 & 0.9 \\
\hline LAMA & 0.9 & 6.7 \\
\hline ICS & 1.8 & 13.7 \\
\hline $\mathrm{LABA}+\mathrm{ICS}$ & 3.8 & 29.4 \\
\hline LABA+LAMA+ICS & 0.6 & 4.8 \\
\hline Theophylline & 0.0 & 0.5 \\
\hline Mucolytics & 0.4 & 1.3 \\
\hline OCS & 0.4 & 1.8 \\
\hline \multicolumn{3}{|l|}{ Comorbidity } \\
\hline Cardiovascular disease ${ }^{\#}$ & 6.0 & 7.0 \\
\hline Diabetes mellitus" & 3.5 & 4.7 \\
\hline Depression $^{+}$ & 13.9 & 21.1 \\
\hline Severe depression & 3.4 & 5.4 \\
\hline
\end{tabular}

Data are presented as weighted mean \pm SD or median (interquartile range) for continuous outcomes and weighted proportion (\%) for categorical outcomes, unless otherwise stated. BMI: body mass index; MET: metabolic equivalent of tasks; FEV1: forced expiratory volume in $1 \mathrm{~s}$; FVC: forced vital capacity; SABA: short-acting $\beta$-agonist; LABA: long-acting $\beta$-agonist; SAMA: short-acting muscarinic antagonist; LAMA: long-acting muscarinic antagonist; ICS: inhaled corticosteroid; OCS: oral corticosteroid. \#: myocardial infarction, angina, congestive heart failure, peripheral vascular disease; ๆ: defined as self-reported diabetes mellitus type 1 or type 2 or medication use; ${ }^{+}$: defined as an Inventory of Depressive Symptomatology score $\geqslant 18$ (severe depression as a score $\geqslant 29$ ). 
TABLE 2 Prevalence of asthma-chronic obstructive pulmonary disease (COPD) overlap syndrome (ACOS) in the total weighted study population and asthma/COPD subpopulation according to different ACOS definitions

Total population Asthma/COPD population

$\begin{array}{lcr}\text { Subjects n } & 5675 & 846 \\ \text { ACOS definition } & & 10.3 \\ \text { 1) COPD AND Asthma in registry } & 1.2 & 10.3 \\ \text { 2) COPD AND Asthma in registry OR ACOS as text in EMR } & 1.2 & 4.4 \\ \text { 3) Self-reported COPD AND Asthma } & 0.5 & 4.7 \\ \text { 4) FEV } 1 / \text { FVC <0.7 AND at least 10 pack-years AND } & 0.6 & 9.2 \\ \text { asthma before } 40 \text { years of age } & & \\ \text { 5) COPD in registry OR Self-reported AND FEV } 1 / F V C<0.7 & 1.1 & 38.3 \\ \text { AND Asthma in registry OR Self-reported } & 4.9 \\ \text { 6) COPD in registry OR Self-reported OR FEV } 1 / F V C<0.7 & & \\ \text { AND Asthma in registry OR Self-reported OR FeNO } & \end{array}$

All values are expressed as weighted proportion (\%), unless otherwise stated. EMR: electronic medical record; FEV1: forced expiratory volume in $1 \mathrm{~s}$; FVC: forced vital capacity; FeNO: exhaled nitric oxide fraction.

text. However, ACOS prevalence was more than halved when ACOS was defined as self-reported compared with the registry-based definition: $0.5 \%$ and $4.4 \%$ versus $1.2 \%$ and $10.3 \%$ in the total and asthma/COPD subpopulation, respectively. When a stricter definition of COPD was used by requiring airway obstruction defined by FEV1/FVC $<0.7$ (definition 5), ACOS prevalence remained similar: $1.2 \%$ versus $1.1 \%$ and $10.3 \%$ versus $9.2 \%$ in the total and asthma/COPD subpopulation, respectively. ACOS prevalence was highest when ACOS was defined by doctor registry or self-reported, FEV1/FVC $<0.7$ or $F$ eNO $\geqslant 45 \mathrm{ppb}$ (definition 6; total population $4.9 \%$; asthma/COPD population $38.3 \%$ )

Agreement between four of the six ACOS definitions is depicted in figure 1. Only one out of $846(0.1 \%)$ patients within the asthma/COPD subpopulation was classified as ACOS by all four definitions. Statistical agreement between different ACOS definitions is shown in table 3. There was maximal agreement (1.00)

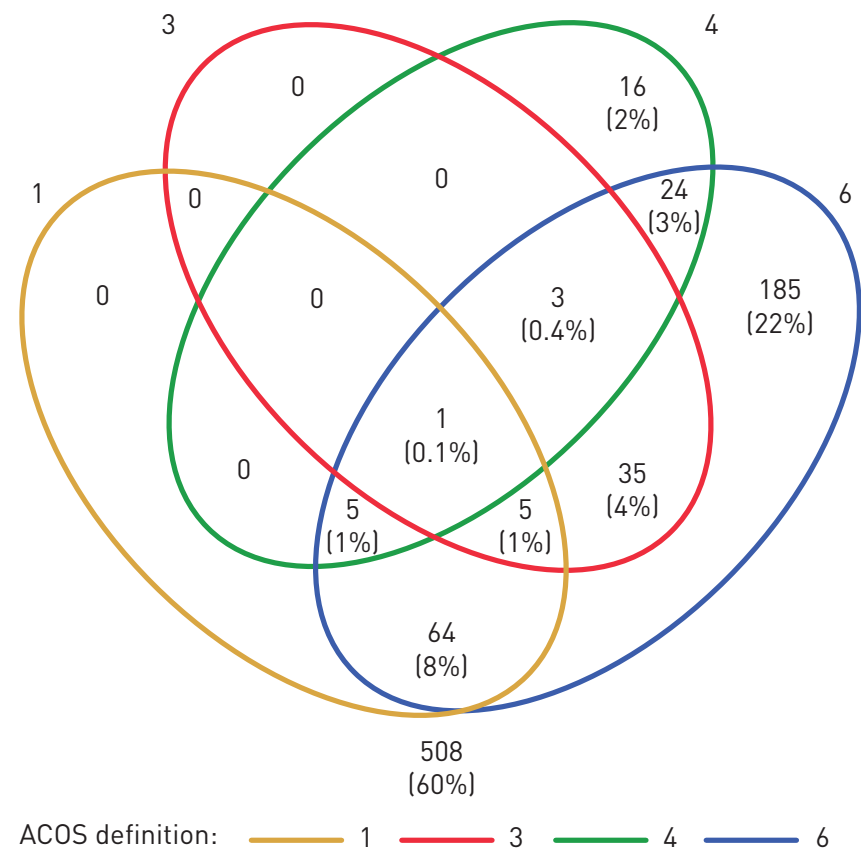

FIGURE 1 Venn diagram of overlap between different asthma-chronic obstructive pulmonary disease (COPD) overlap syndrome (ACOS) definitions (see main text and table 2) within the asthma/COPD subpopulation. Only one out of $846(0.1 \%)$ patients was classified as ACOS by all four definitions. 
TABLE 3 Agreement ( $\kappa$ ) between different definitions of asthma-chronic obstructive pulmonary disease (COPD) overlap syndrome (ACOS) patients in the asthma/COPD study population\#

\begin{tabular}{|c|c|c|c|c|c|c|}
\hline \multirow[t]{2}{*}{ ACOS Definition $\pi$} & \multicolumn{6}{|c|}{ ACOS Definition? } \\
\hline & 1 & 2 & 3 & 4 & 5 & 6 \\
\hline 1 & 1.00 & & & & & \\
\hline 2 & 1.00 & 1.00 & & & & \\
\hline 3 & 0.04 & 0.04 & 1.00 & & & \\
\hline 4 & 0.03 & 0.03 & 0.03 & 1.00 & & \\
\hline 5 & 0.41 & 0.41 & 0.23 & 0.25 & 1.00 & \\
\hline 6 & 0.27 & 0.27 & 0.16 & 0.09 & 0.28 & 1.00 \\
\hline
\end{tabular}

between registry-based ACOS (definition 1) and registry-based plus EMR text (definition 2), because no additional ACOS cases were identified in the EMR text. However, agreement between registry-based (definition 1) and self-reported ACOS (definition 3) was only 0.04. This increased to 0.41 when lung function $(\mathrm{FEV} 1 / \mathrm{FVC}<0.7)$ was added in definition 5.

\section{Differences in patient characteristics across ACOS definitions}

When ACOS was defined based on registry or self-report, only $51 \%$ and $33 \%$ of the patients, respectively, had FEV1/FVC $<0.7$ (table 4). Patient characteristics were similar across ACOS definitions, except for short-acting $\beta$-agonist use and asthma duration. The use of short-acting $\beta$-agonists was significantly higher when ACOS was defined by the three major consensus criteria (definition 1 versus 4: 15\% versus 42\%; odds ratio 4.2, 95\% CI 1.1-15.8). Compared with registry-based ACOS (definition 1), asthma duration was longer in patients with self-reported ACOS (definition 3 versus 1: 45 versus 11 years; mean difference 22 years, 95\% CI 12-33 years) and ACOS defined by three major consensus criteria (definition 4 versus 1 : mean difference 25 years, 95\% CI 19-32 years).

\section{Exacerbation rates}

During a median (IQR) follow-up time of 1.8 (1.2-3.0) years, a total of 677 exacerbations occurred in the asthma and/or COPD subpopulation $(n=846)$. Mean \pm SD number of exacerbations per year ranged from $0.33 \pm 0.54$ for definition 5 to $0.56 \pm 0.81$ for definitions 1 and 2 (supplementary table S2). The proportion of patients with at least one exacerbation per year was highest when ACOS was defined by registry codes (22.7\%; supplementary table S2). Exacerbation risk of ACOS patients compared with asthma and COPD patients is depicted in figure 2 and supplementary tables S3 and S4. Compared with asthma patients, exacerbation risk was higher in ACOS patients. The highest exacerbation risk was observed when ACOS was defined by registry codes (asthma versus ACOS definition 1: adjusted IRR 1.6, 95\% CI 1.2-2.1). Compared with COPD patients, ACOS patients across all definitions were not at higher risk for exacerbations. Adjusted IRRs ranged from 0.5 (definition 5; 95\% CI 0.4-0.7) to 0.9 (definition 1; 95\% CI $0.7-1.1)$.

\section{Discussion}

The aim of this study was to study the agreement between different ACOS definitions, and to assess the impact of these definitions on prevalence, patient characteristics and clinical outcomes. The main findings of this study are that 1) the prevalence of ACOS strongly depends on its definition, 2) the agreement between definitions was low, 3) patient characteristics across ACOS definitions are similar, except for asthma duration, and 4) exacerbation rates of ACOS patients were only higher than patients with asthma only, but only with the registry-based ACOS definition.

\section{Comparison with previous findings}

The prevalence of ACOS in general population cohorts varies from $1.8 \%$ to $4.5 \%$ in the current literature $[10,23]$. In our study, the age range was $45-65$ years. In the same age range, a prevalence of $2.1 \%$ was found in an Italian cohort with ACOS defined as doctor-diagnosed COPD and asthma [24], while a lower prevalence (1.2\%; table 2 ) was found in our study with the same ACOS definition. This difference may be caused by the higher proportion of current smokers in the Italian cohort compared with our cohort (23.4\% versus $16.0 \%$ ), because continuous smoking could lead to irreversible obstruction in asthma patients and subsequent diagnosis of ACOS. The prevalence of ACOS was lowest in our general population 
TABLE 4 Characteristics of asthma-chronic obstructive pulmonary disease (COPD) overlap syndrome (ACOS) patients in the asthma/COPD study population" according to different ACOS definitions

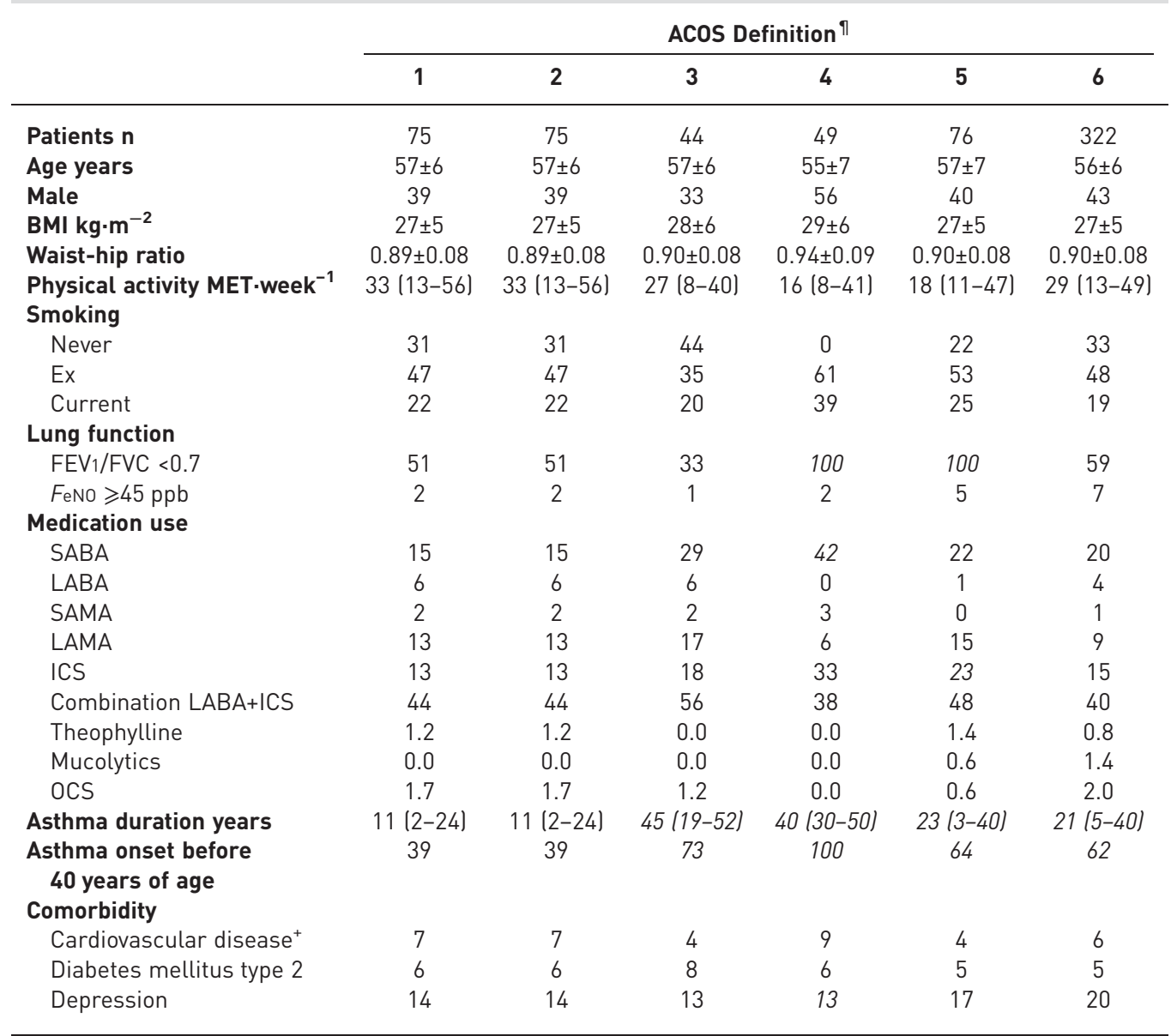

Data are presented as weighted mean \pm SD or median (interquartile range) for continuous outcomes and weighted proportion (\%) for categorical outcomes, unless otherwise stated. BMI: body mass index; MET: metabolic equivalent of tasks; FEV1: forced expiratory volume in $1 \mathrm{~s}$; FVC: forced vital capacity; FeNO: exhaled nitric oxide fraction; SABA: short-acting $\beta$-agonist; LABA: long-acting $\beta$-agonist; SAMA: short-acting muscarinic antagonist; LAMA: long-acting muscarinic antagonist; ICS: inhaled corticosteroid; OCS: oral corticosteroid. " infarction, angina, congestive heart failure, peripheral vascular disease. Statistically significant differences are given in italics.

cohort when ACOS was defined by self-reporting. A previous general population cohort in US participants reported an ACOS prevalence of $2.4 \%$ with self-report [25], which is a factor of 5 higher than our prevalence using this definition $(0.5 \%$; table 2$)$. This difference is probably partly caused by the definitions of asthma used: in our study, participants were asked to report current asthma, while in this previous study former asthma was also included in the definition of self-reported ACOS [25]. As current asthma is highest in young age groups and we included middle-aged subjects (45-65 years), this may have reduced our self-reported ACOS prevalence. Another explanation for the low prevalence we found could be that the population aged $>65$ years was not included in our cohort. Previous studies found that ACOS prevalence increases with age $[23,24]$. It is therefore likely that the prevalence of ACOS in the present study is an underestimation. Still, our middle-aged population represents a patient group in which the differentiation between asthma, COPD and ACOS is a larger clinical challenge than in young or very old age groups. Finally, the estimated prevalence of asthma and COPD in our population could be an underestimation of true disease prevalence, because we used doctor registries and patient questionnaires. Previous studies showed that, in a population aged $\geqslant 45$ years, only $25-50 \%$ of patients with chronic airflow obstruction are known (and thus registered) to their doctor [26]. However, we found that, when ACOS was defined by doctor registries or self-report, a high number of patients did not show an 

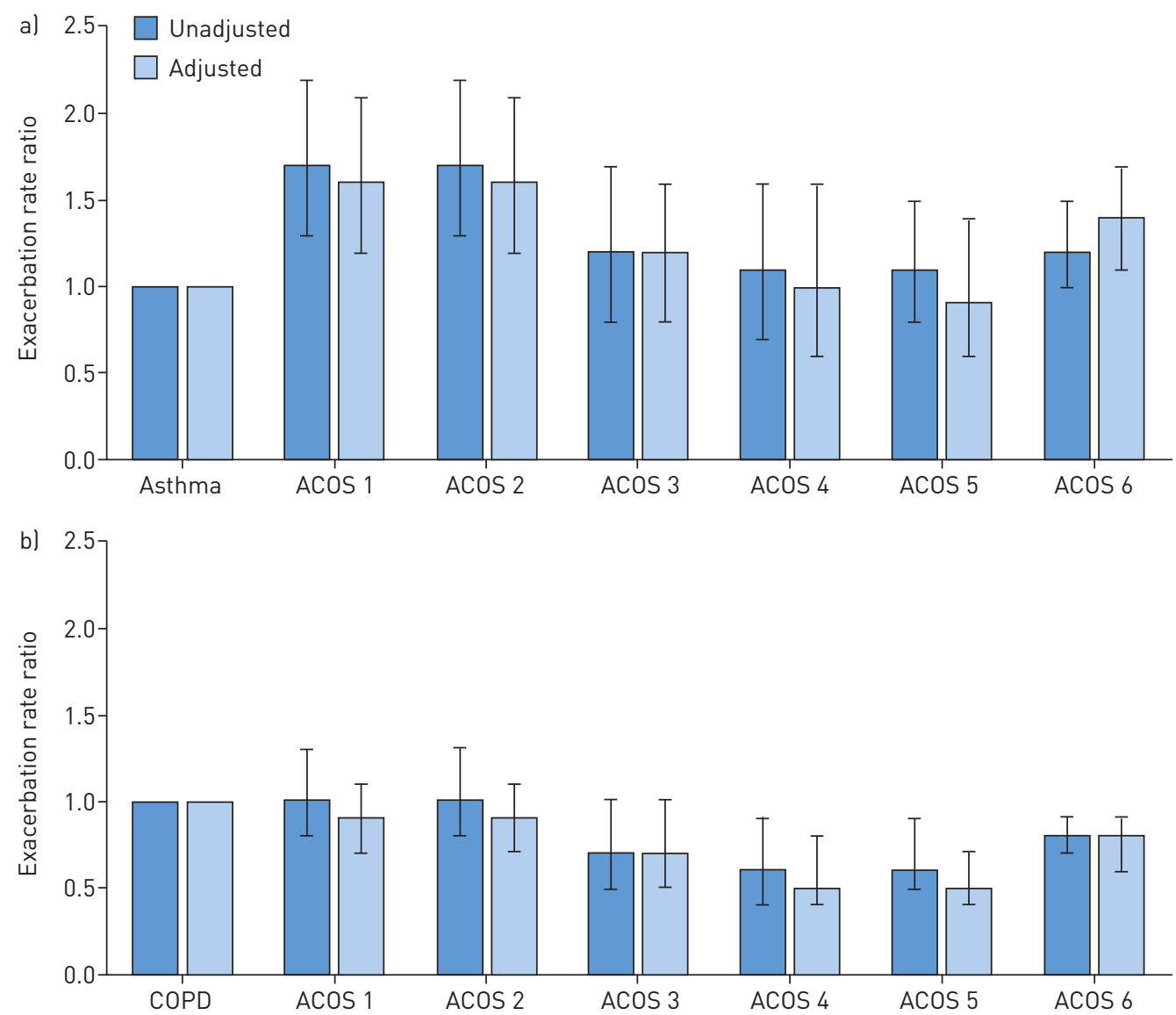

FIGURE 2 Exacerbation rate ratio (95\% Cl) of asthma-chronic obstructive pulmonary disease (COPD) overlap syndrome (ACOS) patients laccording to different definitions; see main text and table 2) compared with patients with only al asthma or b) COPD. Unadjusted analyses, and adjusted analyses for age, sex, body mass index, current smoking, forced expiratory volume in $1 \mathrm{~s} /$ forced vital capacity ratio at baseline, inhaled corticosteroid use at baseline and the number of exacerbations in the year before baseline.

obstructive pattern at pulmonary function testing. In our study, only $51 \%$ of the patients who had a co-diagnosis of asthma and COPD in their EMR had FEV1/FVC $<0.7$. This is in line with a previous study in which only $50 \%$ met the GOLD criteria for COPD in general practice [27]. Another interesting finding of our study is that asthma duration was longer with self-reported ACOS. This suggests that patients with early-onset asthma are specifically identified by self-reporting. A recent study also showed the clinical importance of the age of asthma onset in ACOS patients: patients with ACOS with asthma onset after 40 years of age had a twofold higher risk of hospital admission for asthma and COPD than ACOS patients with asthma onset before 40 years of age [28]. This is in line with our study, in which we found a higher risk of exacerbations in the ACOS group with late-onset asthma (ACOS definition 1) compared with young-age asthma onset (ACOS definition 3; figure 2a).

To the best of our knowledge, this is the first study to compare the agreement between different ACOS definitions in one study population. Most importantly, we observed a low agreement $(\kappa=0.04)$ between the two most used definitions in the current ACOS literature: doctor-diagnosed ACOS based on EMR codes and self-reported ACOS [10]. This impedes the comparability of outcomes across studies. Although clinical characteristics of patients were similar between registry-based and self-reported ACOS, we observed a difference in prevalence and exacerbation rate between these definitions. This could have implications for the identification and treatment of ACOS patients in clinical practice, as discussed later in the section on "Clinical implications and future directives".

In the present study, exacerbation rates of asthma, COPD and ACOS patients were low. However, these rates are not unexpected because we studied a low-risk general population cohort and these rates are in line with a previous general population study [29]. In our study, exacerbation rates of registry-based ACOS patients were higher compared with asthma patients, which is in line with the current literature [13]. However, in this study we did not observe a higher risk for exacerbations of ACOS patients compared with 
COPD patients. This is in line with a recent study in which ACOS patients did not show a higher exacerbation rate compared with COPD patients [30], but is in contrast with most previous studies, which reported a higher risk of exacerbations in ACOS patients compared with COPD patients [24, 29, 31-33]. However, most of these studies used a more stringent definition of COPD (post-bronchodilator FEV1/FVC $<0.7$ ) than we did (only COPD registry code), resembling a higher-risk COPD population. Furthermore, exacerbations in these studies were self-reported, while we used prescription of medication to define exacerbations. Thus, we only reported outcomes on moderate exacerbations, whereas previous studies also included self-reported exacerbations for which no medication was prescribed (mild) and hospitalisation (severe exacerbations) [34]. If exacerbation severity would differentially affect COPD and ACOS patients, this could explain the absence of risk difference for moderate exacerbations as suggested by our study.

\section{Strengths and limitations}

A major strength of this study is that, to the best of our knowledge, it is the first to compare different ACOS definitions within one study population. This is of major importance to this field, because ACOS definitions vary among studies, which makes comparison between studies difficult. This study sheds light on the comparability of patient characteristics and exacerbations between studies using different definitions. Another strength of the current study is the prospective collection of baseline characteristics and exacerbations during follow-up. We were able to use real-life medical records to define ACOS, asthma and COPD, which improves generalisability and translation of our results into clinical practice. Previous studies used self-reported exacerbations in the year before baseline as outcome [24, 29, 31-33]. Furthermore, our use of real-life medication prescription data implies high generalisability to the general population. For example, bronchodilator medication use in asthma and COPD patients was similar in our cohort compared with previous general population cohorts in which ACOS was studied $[29,35]$.

A limitation is that we were not able to differentiate between moderate exacerbations (medication prescription) and severe exacerbations (hospital admission). Furthermore, we did not register self-initiated exacerbation medication by patients. Therefore, our definition of an exacerbation could have led to an underestimation of exacerbation rates. However, the degree of underestimation would be the same in all groups we compared and would thus not lead to another direction of our conclusions with respect to differences in exacerbation risk between the groups we compared. Still, future studies that include self-registered and severe exacerbations as an outcome could provide further insight. Another limitation is that we did not include blood or sputum eosinophils or IgE to define ACOS, although their use is suggested in ACOS guidelines and recent consensus criteria [9]. However, our definitions include clinical characteristics which are easy to collect from patients. Furthermore, we included FeNO in definition 6, which correlates with blood eosinophilia and is used to define ACOS in the Czech guideline [6, 36]. Similarly, we were not able to use asthma symptoms at the moment of diagnosis or family history to define ACOS, because this was not included in the baseline questionnaire. Finally, our sample sizes in some subgroups of ACOS definitions were small, which may affect the analysis of possible differences between ACOS definitions. However, our sample size resembles previous important ACOS studies in the general population [29, 33]. Future studies with larger sample sizes in certain ACOS subgroups could investigate these findings more closely.

\section{Clinical implications and future directives}

Currently, definitions of ACOS vary across scientific studies and do not follow the available practice guidelines. This discrepancy may be reduced by simplifying the definition of ACOS in primary care and using a stepwise strategy to diagnose ACOS in secondary care. In line with a previous study, we suggest to identify presumptive ACOS patients in primary care by using the co-registration of asthma and COPD in the electronic record or asking COPD patients about their asthma history [37]. Subsequently, general practitioners could refer presumptive ACOS patients for diagnostic workup in secondary care. In secondary care, endotypic and phenotypic characterisation of ACOS patients should be performed. In our opinion, describing patient characteristics and personalising treatment accordingly is preferable above diagnosing or not diagnosing patients with ACOS according to one stringent definition [38, 39]. This stepped-care approach also could benefit future research in ACOS patients. For example, research on new treatments in a heterogeneous and not well-defined patient group could lead to negative results for the whole group, although subgroups could benefit from this treatment. Thus, when designing and selecting ACOS patients for future clinical trials, extensive clinical phenotyping will help to determine important subgroups and personalise treatment $[13,39]$. Future studies should also address other relevant outcomes in addition to exacerbations, such as quality of life and respiratory symptoms. Finally, the ACOS GINA guideline suggests to start with ICS in ACOS patients with features of asthma, although evidence about the risk-benefit of ICS in ACOS patients is currently lacking and should be of interest for future studies. 


\section{Conclusions}

This study adds important knowledge about the agreement between different ACOS definitions and their relation with clinical outcomes, and thus helps to compare ACOS studies. Given the low agreement between definitions, differences in prevalence, patient characteristics and risk of exacerbations, consensus about the definition of ACOS in different care settings is urgently needed.

\section{Acknowledgements}

We express our gratitude to all individuals who participated in the NEO study. We are grateful to all participating general practitioners for inviting eligible participants. We furthermore thank all research nurses for collecting the data and Ingeborg de Jonge (Dept of Clinical Epidemiology, LUMC, Leiden, The Netherlands) for data management of the NEO study.

\section{References}

1 Global Initiative for Chronic Obstructive Lung Disease. Global Strategy for the Diagnosis, Management and Prevention of COPD. 2016. http://goldcopd.org/global-strategy-diagnosis-management-prevention-copd-2016 Date last accessed: October 10, 2016.

2 Global Initiative for Asthma. Global Strategy for Asthma Management and Prevention. 2016. http://ginasthma.org/ 2016-gina-report-global-strategy-for-asthma-management-and-prevention Date last accessed: October 10, 2016.

3 Global Initiative for Asthma. Diagnosis of Diseases of Chronic Airflow Limitation: Asthma, COPD and AsthmaCOPD Overlap Syndrome (ACOS). 2015. http://ginasthma.org/asthma-copd-and-asthma-copd-overlap-syndromeacos Date last accessed: October 10, 2016.

4 Gibson PG, Simpson JL. The overlap syndrome of asthma and COPD: what are its features and how important is it? Thorax 2009; 64: 728-735.

5 Committee for the Third Edition of the COPD Guidelines of The Japanese Respiratory Society. Guidelines for the Diagnosis and Treatment of COPD (Chronic Obstructive Pulmonary Disease). 3rd Edn. 2010. www.jrs.or.jp/ uploads/uploads/files/photos/765.pdf Date last accessed: October 10, 2016.

6 Koblizek V, Chlumsky J, Zindr V, et al. Chronic Obstructive Pulmonary Disease: official diagnosis and treatment guidelines of the Czech Pneumological and Phthisiological Society; a novel phenotypic approach to COPD with patient-oriented care. Biomed Pap Med Fac Univ Palacky Olomouc Czech Repub 2013; 157: 189-201.

7 National Asthma Council Australia. Australian Asthma Management Handbook. 2016. www.asthmahandbook.org.au Date last accessed: October 10, 2016

8 Soler-Cataluna JJ, Cosio B, Izquierdo JL, et al. Consensus document on the overlap phenotype COPD-asthma in COPD. Arch Bronconeumol 2012; 48: 331-337.

9 Sin DD, Miravitlles M, Mannino DM, et al. What is asthma-COPD overlap syndrome? Towards a consensus definition from a round table discussion. Eur Respir J 2016; 48: 664-673.

10 Nielsen M, Barnes CB, Ulrik CS. Clinical characteristics of the asthma-COPD overlap syndrome - a systematic review. Int J Chron Obstruct Pulmon Dis 2015; 10: 1443-1454.

11 Loukides S, Bakakos P, Kostikas K. The asthma-COPD overlap syndrome: ACOS, CAOS or CHAOS? Pneumon 2014; 27: 303-305

12 Pavord I, Bush A. Two lovely black eyes; oh, what a surprise! Thorax 2015; 70: 609-610.

13 Slats A, Taube C. Asthma and chronic obstructive pulmonary disease overlap: asthmatic chronic obstructive pulmonary disease or chronic obstructive asthma? Ther Adv Respir Dis 2016; 10: 57-71.

14 de Mutsert R, den Heijer M, Rabelink TJ, et al. The Netherlands Epidemiology of Obesity (NEO) study: study design and data collection. Eur J Epidemiol 2013; 28: 513-523.

15 Wendel-Vos GC, Schuit AJ, Saris WH, et al. Reproducibility and relative validity of the short questionnaire to assess health-enhancing physical activity. J Clin Epidemiol 2003; 56: 1163-1169.

16 Rush AJ, Gullion CM, Basco MR, et al. The Inventory of Depressive Symptomatology (IDS): psychometric properties. Psychol Med 1996; 26: 477-486.

17 Schulte-van Maaren YW, Carlier IV, Zitman FG, et al. Reference values for major depression questionnaires: the Leiden Routine Outcome Monitoring Study. J Affect Disord 2013; 149: 342-349.

18 Miller MR, Hankinson J, Brusasco V, et al. Standardisation of spirometry. Eur Respir J 2005; 26: 319-338.

19 Burge S, Wedzicha JA. COPD exacerbations: definitions and classifications. Eur Respir J Suppl 2003; 41: 46s-53s.

20 Schneider A, Faderl B, Schwarzbach J, et al. Prognostic value of bronchial provocation and FENO measurement for asthma diagnosis - results of a delayed type of diagnostic study. Respir Med 2014; 108: 34-40.

21 Korn EL, Graubard BI. Epidemiologic studies utilizing surveys: accounting for the sampling design. Am J Public Health 1991; 81: 1166-1173.

22 Centraal Bureau voor de Statistiek. Uitkomsten Gezondheidsmonitor 2012. www.cbs.nl/nl-nl/maatwerk/2013/37/ uitkomsten-gezondheidsmonitor-2012 Date last accessed: October 10, 2016.

23 Gibson PG, McDonald VM. Asthma-COPD overlap 2015; now we are six. Thorax 2015; 70: 683-691.

24 de Marco R, Pesce G, Marcon A, et al. The coexistence of asthma and chronic obstructive pulmonary disease (COPD): prevalence and risk factors in young, middle-aged and elderly people from the general population. PLoS One 2013; 8: e62985.

25 Pleasants RA, Ohar JA, Croft JB, et al. Chronic obstructive pulmonary disease and asthma-patient characteristics and health impairment. COPD 2014; 11: 256-266.

26 van Schayck CP, Chavannes NH. Detection of asthma and chronic obstructive pulmonary disease in primary care. Eur Respir J Suppl 2003; 39: 16s-22s.

27 Sichletidis L, Chloros D, Spyratos D, et al. The validity of the diagnosis of chronic obstructive pulmonary disease in general practice. Prim Care Respir J 2007; 16: 82-88.

28 Lange P, Çolak Y, Ingebrigtsen TS, et al. Long-term prognosis of asthma, chronic obstructive pulmonary disease, and asthma-chronic obstructive pulmonary disease overlap in the Copenhagen City Heart study: a prospective population-based analysis. Lancet Respir Med 2016; 4: 454-462. 
29 Menezes AM, Montes de Oca M, Pérez-Padilla R, et al. Increased risk of exacerbation and hospitalization in subjects with an overlap phenotype: COPD-asthma. Chest 2014; 145: 297-304.

30 Cosio BG, Soriano JB, López-Campos JL, et al. Defining the asthma-COPD overlap syndrome in a COPD cohort. Chest 2016; 149: 45-52.

31 Izquierdo-Alonso JL, Rodriguez-Gonzálezmoro JM, de Lucas-Ramos P, et al. Prevalence and characteristics of three clinical phenotypes of chronic obstructive pulmonary disease (COPD). Respir Med 2013; 107: 724-731.

32 Hardin M, Silverman EK, Barr RG, et al. The clinical features of the overlap between COPD and asthma. Respir Res 2011; 12: 127.

33 Miravitlles M, Soriano JB, Ancochea J, et al. Characterisation of the overlap COPD-asthma phenotype. Focus on physical activity and health status. Respir Med 2013; 107: 1053-1060.

34 Rodriguez-Roisin R. Toward a consensus definition for COPD exacerbations. Chest 2000; 117: 5 Suppl. 2, 398S-401S.

35 Chung WS, Lin CL, Kao CH. Comparison of acute respiratory events between asthma-COPD overlap syndrome and COPD patients: a population-based cohort study. Medicine 2015; 94: e755.

36 Schleich FN, Seidel L, Sele J, et al. Exhaled nitric oxide thresholds associated with a sputum eosinophil count $\geqslant 3 \%$ in a cohort of unselected patients with asthma. Thorax 2010; 65: 1039-1044.

37 Barrecheguren M, Roman-Rodriguez M, Miravitlles M. Is a previous diagnosis of asthma a reliable criterion for asthma-COPD overlap syndrome in a patient with COPD? Int J Chron Obstruct Pulmon Dis 2015; 10: 1745-1752.

38 Bateman ED, Reddel HK, van Zyl-Smit RN, et al. The asthma-COPD overlap syndrome: towards a revised taxonomy of chronic airways diseases? Lancet Respir Med 2015; 3: 719-728.

39 Cazzola M, Rogliani P. Do we really need asthma-chronic obstructive pulmonary disease overlap syndrome? J Allergy Clin Immunol 2016; 138: 977-983. 